\title{
PRODUÇÃO DE SERRAPILHERIA EM BIOMAS NO ESTADO DE MATO GROSSO
}

\author{
Carla Maria Abido Valentini ${ }^{1}$ \\ Luciana Sanches ${ }^{2}$ \\ Osvaldo Borges Pinto Junior ${ }^{3}$ \\ Rozilaine Aparecida Pelegrine Gomes de Faria ${ }^{4}$
}

\section{INTRODUÇÃO}

\section{Biomas no estado de Mato Grosso e suas transformações}

O Estado de Mato Grosso, localizado na área central da América do Sul, engloba em seu território a biodiversidade de três grandes biomas brasileiros: a Amazônia, o Cerrado e o Pantanal mato-grossenses. Contudo, essas formações biogeográficas e seus ecótonos têm sofrido modificações constantes pela pressão antrópica (CAMARGO et al., 2014; NUNES et al., 2017).

De acordo com Neuburger (2003), a incorporação definitiva de Mato Grosso à economia nacional se deu somente nos anos 70 do século XX, iniciada pela implantação de uma rede rodoviária, pelos baixos preços da terra, pela colonização particular por empresas sulistas e pela migração crescente de colonos do sul do país. Com isso, áreas enormes foram desmatadas, deslocando e expulsando os grupos tradicionais e indígenas.

As áreas nativas do Cerrado vêm sendo removidas em um ritmo acelerado e desordenado, sendo substituídas pela produção agrícola mecanizada e pelos extensos latifúndios de gado de

1 Professora Doutora do Instituto Federal de Educação, Ciência e Tecnologia de Mato Grosso, campus Cuiabá-BelaVista. carla.valentini@blv.ifmt.edu.br

2 Professora Doutora do Depto de Engenharia Sanitária e Ambiental da Universidade Federal de Mato Grosso. Isanches@hotmail.com

3 Professor Doutor da Universidade de Cuiabá. osvaldo.borges@kroton.com.br

4 Professora Doutora do Instituto Federal de Educação, Ciência e Tecnologia de Mato Grosso, campus Cuiabá-Bela Vista. rozilaine.faria@blv.ifmt.edu.br 
corte. Tornou-se a principal região brasileira produtora de grãos (AGUIAR \& CAMARGO, 2004), o que lhe valeu a alcunha de "celeiro do mundo".

O plantio, especialmente de soja, que começou nos cerrados centrais, aumentou $400 \%$ nos últimos dez anos e migrou para cerca de 500 quilômetros ao norte, deslocando a fronteira agrícola. Por conseguinte, a Amazônia mato-grossense sofreu desmatamento e queimadas tanto para o avanço da fronteira agrícola como para a pecuária e a exploração de madeiras (DOMINGUES \& BERMANN; 2012; NUNES et al., 2017).

A expansão desordenada das atividades agropecuárias também não poupou o Pantanal mato-grossense: tem contribuído com a intensificação do assoreamento, a contaminação dos rios com biocidas e principalmente com a perda da biodiversidade, além da degradação dos ambientes naturais da região (SANTOS, 2006). Ademais, ela tem sofrido modificações na geometria hidráulica dos seus rios devido ao desmatamento do planalto, que drena para o Pantanal, assim como pela construção de represas para o fornecimento de energia elétrica (NUNES et al., 2017).

Essas modificações causadas pela ação antrópica podem desencadear uma série de alterações nos meios naturais em equilíbrio. Por mais que o conceito de estabilidade ecossistêmica não seja unânime, visto que depende das escalas espaciais e temporais adotadas (MITCHELL et al., 2000), a perda da diversidade de espécies pode diminuir a estabilidade dos ecossistemas (McCANN, 2000). Ocorrem, então, os estágios sucessionais, e permanecem nos ambientes as espécies que melhor se adaptam às competições por espaço e nutrientes. Toda essa dinâmica promove o aporte de serrapilheira sobre o solo. Ela cria condições aos decompositores ao promover a retenção de umidade, quantidade e qualidade de nutrientes (SOUSA, 2015).

Existem vários métodos para caracterizar e quantificar as respostas a tensões ambientais. Um deles consiste em coletar e quantificar o material decíduo aportado ao solo, a serrapilheira, visto que a maior parte da produção de energia e nutrientes é direcionada à própria manutenção da funcionalidade e estrutura das espécies (CLARK et al., 2001; MONTEZUMA, 2005; ABREU, 2006; SCORIZA et al., 2012). 
A serrapilheira pode ser classificada como um bioindicador de reação, uma vez que responde, em função de alterações no meio, com mudanças nos seus processos de deposição (KLUMPP, 2001). 0 material decíduo ao longo do ano permite estimar a produção anual por hectare, informação relevante por possibilitar a comparação com outros estudos. Objetiva-se, com isso, verificar se a floresta está se recuperando, visto que a ciclagem de nutrientes se inicia com a deposição da serrapilheira (SIMONELLI et al., 2011). Assim, ao analisar resultados de pesquisas de produção de serrapilheira nos biomas de Mato Grosso e seus ecótonos, de modo a discutir o quantitativo de biomassa de acordo com as condições ambientais apresentadas constitui o objeto científico em questão.

\section{Produção de serrapilheira}

Ecossistemas tropicais, incluindo floresta tropical, florestas sazonais secas, cerrados e áreas inundáveis, desempenham um papel fundamental no estoque e ciclo global de carbono (GRACE et al., 2001; KELLER et al., 2004). Em termos globais, os estoques de carbono no solo são 2-3 vezes maiores que aqueles acima dele (POST et al., 1982; SCHLESINGER, 1991).

A serrapilheira, serapilheira, folhedo ou liteira (do inglês litter) é um importante componente no ecossistema florestal. Caracteriza-se como o material precipitado ao solo pela biota, o que inclui principalmente folhas, galhos, frutos, flores, raízes e resíduos animais (DIAS \& OLIVEIRA FILHO, 1997). Ela representa o maior caminho biológico da transferência de elementos da vegetação para o solo (XU \& HIRATA, 2002), uma vez que, segundo Aerts (1997), aproximadamente $70 \%$ do fluxo anual total de carbono é derivado da decomposição da liteira (acima e abaixo do solo). Sua produção é influenciada por fatores como clima, fertilidade do solo, composição de espécies, estrutura da vegetação e estágio sucessional da floresta (DELITTI, 1995).

Estudos sobre a produção de serrapilheira têm sido feitos por diversos autores em diferentes localidades geográficas. Porém, a comparabilidade de dados tem sido um obstáculo importante, (DIAS \& OLIVEIRA FILHO, 1997) devido às diferentes metodologias aplicadas e aos distintos comportamentos dos ecossistemas (SILVA et al., 2007). Outro obstáculo apontado por Vidal et al. (2007) é a 
fragmentação dos habitats. Ela leva à formação de manchas remanescentes, chamadas de fragmentos. Seus tamanhos são variáveis e ficam isolados de outros fragmentos semelhantes, em maior ou menor grau. $\mathrm{O}$ tamanho e o grau de isolamento desses fragmentos podem afetar a produção de liteira. Por fim, é necessário considerar que a criação dos fragmentos implica na criação de uma borda, ou seja, uma região de contato entre a área ocupada com agricultura ou pasto e o fragmento de floresta, que normalmente não é considerada nos estudos (RODRIGUES, 1998).

Silva et al. (2007) observaram a importância de estudos que busquem comparar a produção de serrapilheira em diferentes biomas regidos pelo mesmo comportamento climático, pois se tornam de grande importância para a compreensão e determinação de modelos regionais.

\section{DELINEAMENTO EXPERIMENTAL}

As coletas de serrapilheira produzida (do inglês, litter fall) são realizadas por meio de coletores confeccionados a critério do pesquisador. Eles podem ser quadrados ou cônicos, de madeira ou metal/plástico e com dimensões que variam de 0,25 a $1 \mathrm{~m}^{2}$ de área (Figura1). Os coletores quadrados possuem uma tela de naylon ${ }^{\circledR}$ na parte inferior e são fixados na área por bases. Na literatura, verificam-se variações no tocante à altura do coletor em relação ao solo, encontrando-se valores desde 10 até $70 \mathrm{~cm}$. A mesma variação também é observada para os coletores cônicos, que podem ter tela de naylon ${ }^{\circledR}$ ou serem revestidos por tecido helanca ${ }^{\circledR}$. Eles são fixados no solo por meio de estacas ou amarrados nas árvores com o auxílio de cordas (SCORIZA et al., 2012).

Na maioria dos trabalhos o período de coleta da serrapilheira produzida é mensal, e ela também pode ser realizada quinzenalmente. As amostras coletadas são acondicionadas em sacolas plásticas e levadas para análise em laboratório para processamento. A serrapilheira é transferida para sacolas de papel (papel kraft) de massa conhecida e identificada. Em seguida, são acondicionadas em estufa de circulação forçada de ar. Na literatura, há variações de 50 a 75 ㅇ e 24 a $72 \mathrm{~h}$ em relação à temperatura e ao tempo de permanência na estufa, respectivamente. Entretanto, em todos os 
estudos o objetivo é atingir a massa seca constante do material. Posteriormente, a serrapilheira é triada em frações, isto é, separada de acordo com a sua composição anatômica (folhas, ramos, material reprodutivo, entre outros) e a pesagem do material é feita em balança de precisão).

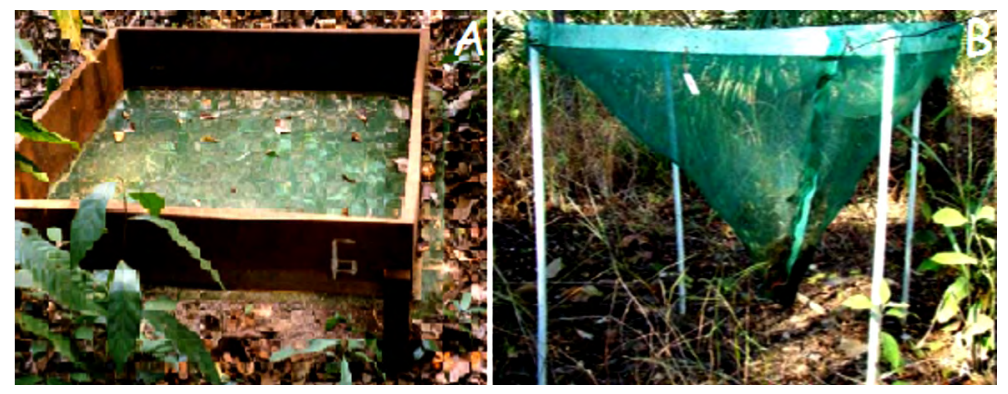

Figura 1. Modelos de coletores de serrapilheira produzida: (A) coletor quadrado (VALENTINI, 2004) e (B) coletor cônico (VALENTINI et al., 2014)

A quantidade e distribuição dos coletores na área dependem dos objetivos e do SCORIZA et al., 2012) delineamento experimental traçado na pesquisa. A quantificação do aporte total de serapilheira é expressa em quantidade por área. Neste trabalho, ela será apresentada em $\mathrm{Mg} \mathrm{ha}{ }^{-1} \mathrm{ano}^{-1}$ (Megagrama por hectare por ano).

\section{Classificação climática e distribuição da vegetação no Estado de Mato Grosso}

Mato Grosso está localizado na região Centro-Oeste do Brasil. É o terceiro maior estado do país, com área total de $903.378 \mathrm{~km}^{2}$, e está compreendido entre as coordenadas $9^{\circ} 27^{\prime} 28^{\prime \prime}$ e $17^{\circ} 50^{\prime}$ 04" S; 50 30' 22" e 61 27' 34" W (IBGE, 2015).

Segundo a classificação de Köppen, o estado de Mato Grosso apresenta dois tipos climáticos: Am, definido como clima tropical de monção e Aw, clima tropical de savana. É uma transição entre o tipo climático Af e Aw e caracteriza-se por apresentar temperatura média superior a 18 ㄷ no mês mais frio e estação seca de pequena duração. O tipo climático Aw, por sua vez, apresenta estação chuvosa no verão (novembro a abril) e estação seca no inverno (maio a outubro), com temperatura média do mês mais frio superior a 18 ㄷ e precipitação do mês mais seco menor que $60 \mathrm{~mm}$ (SOARES et al., 2015). 
A posição geográfica, associada a fatores climáticos, geológicos, pedológicos, fitoecológicos, geomorfológicos e hídricos, condiciona uma grande complexidade ambiental ao Estado de Mato Grosso. Nele se sucedem, no eixo espacial sul-norte, o Complexo do Pantanal no sul, as formações savânicas na região centro-sul, uma variedade de ambientes de transição ecológica na região central e, finalmente, as formações amazônicas no norte (SOUZA et al., 2013).

\section{RESULTADOS E DISCUSSÃO}

Este trabalho faz um levantamento das publicações relacionadas à produção de serrapilheira nos biomas mato-grossenses e seus ecótonos. Os dados que serão apresentados nas Tabelas 1, 2 e 3 foram obtidos por meio da revisão de artigos, dissertações e teses. As localizações dos estudos referenciados nas Tabelas são apresentadas na Figura 2.

Na Tabela abaixo são apresentados os dados obtidos no Cerrado de Mato Grosso.

\begin{tabular}{|c|c|c|c|c|c|}
\hline Município & $\begin{array}{l}\text { Tipologia/ } \\
\text { Região ou } \\
\text { área }\end{array}$ & $\begin{array}{l}\text { Coordenada } \\
\text { geográfica }\end{array}$ & $\begin{array}{c}\text { Produção } \\
\text { serapilheira } \\
\left(\mathrm{Mgha}^{-1} \mathrm{ano}^{-1}\right)\end{array}$ & $\begin{array}{c}\text { Ano da } \\
\text { pesquisa }\end{array}$ & Referência \\
\hline $\begin{array}{l}\text { Nova } \\
\text { Xavantina }\end{array}$ & $\begin{array}{l}\text { Cerrado } \\
\text { stricto } \\
\text { sensu/ } \\
\text { Reserva } \\
\text { Biológica } \\
\text { Municipal } \\
\text { Mário } \\
\text { Viana* }\end{array}$ & 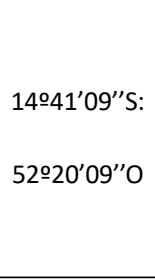 & 0,62 & $\begin{array}{c}2002 a \\
2003\end{array}$ & $\begin{array}{l}\text { Silva et } \\
\text { al.(2007) }\end{array}$ \\
\hline $\begin{array}{l}\text { Nova } \\
\text { Xavantina }\end{array}$ & $\begin{array}{l}\text { Cerradão/ } \\
\text { Reserva } \\
\text { Biológica } \\
\text { Municipal } \\
\text { Mário } \\
\text { Viana* }\end{array}$ & $\begin{array}{l}\text { 14ㄴ109"S: } \\
52 \div 20^{\prime} 09^{\prime \prime O}\end{array}$ & 1,05 & $\begin{array}{c}2002 a \\
2003\end{array}$ & $\begin{array}{l}\text { Silva et } \\
\text { al.(2007) }\end{array}$ \\
\hline $\begin{array}{l}\text { Tangará da } \\
\text { Serra }\end{array}$ & $\begin{array}{l}\text { Fragmento } \\
\text { de } \\
\text { Cerradão/ } \\
\text { Rodovia } \\
\text { MT } 358\end{array}$ & 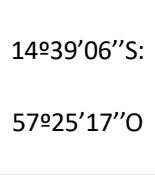 & 2,22 & $\begin{array}{c}2005 a \\
2006\end{array}$ & $\begin{array}{l}\text { Maman et } \\
\text { al.(2007) }\end{array}$ \\
\hline
\end{tabular}

Continua... 


\begin{tabular}{|c|c|c|c|c|c|}
\hline Cuiabá & $\begin{array}{l}\text { Área } \\
\text { revegetada } \\
\text { de } \\
\text { Cerrado/ } \\
\text { Parque } \\
\text { Estadual } \\
\text { Massairo } \\
\text { Okamura }\end{array}$ & $\begin{array}{l}15034^{\prime} 45,02^{\prime \prime} \mathrm{S}: \\
56003^{\prime} 45,78^{\prime \prime} \mathrm{O}\end{array}$ & 11,89 & $\begin{array}{c}2009 \text { a } \\
2011\end{array}$ & $\begin{array}{l}\text { Valentini et } \\
\text { al. (2014) }\end{array}$ \\
\hline $\begin{array}{l}\text { Nova } \\
\text { Xavantina }\end{array}$ & $\begin{array}{l}\text { Cerrado } \\
\text { típico/ } \\
\text { Parque } \\
\text { Municipal } \\
\text { Bacaba }\end{array}$ & $\begin{array}{l}14^{\circ} 42^{\prime} 02,3^{\prime \prime} \mathrm{S}: \\
52^{\circ} 21^{\prime} 02,6^{\prime \prime} \mathrm{O}\end{array}$ & 4,06 & $\begin{array}{c}2011 a \\
2013\end{array}$ & $\begin{array}{l}\text { Valadão et } \\
\text { al.(2016) }\end{array}$ \\
\hline Cuiabá & $\begin{array}{l}\text { Cerrado } \\
\text { típico/ } \\
\text { Parque } \\
\text { Estadual } \\
\text { Mãe } \\
\text { Bonifácia }\end{array}$ & $\begin{array}{l}\text { 153'44"S: } \\
5805^{\prime} 16^{\prime \prime O}\end{array}$ & 6,90 & $\begin{array}{c}2015 a \\
2016\end{array}$ & $\begin{array}{l}\text { Pereira } \\
\text { (2017) }\end{array}$ \\
\hline
\end{tabular}

TABELA 1- Produção de serrapilheira no Cerrado de Mato Grosso

A Reserva Biológica Municipal Mário Viana é o atual Parque Municipal Bacaba

Na Tabela abaixo são apresentados os dados obtidos na Floresta Amazônica de Mato Grosso.

\begin{tabular}{|c|c|c|c|c|c|}
\hline Município & $\begin{array}{l}\text { Tipologia/ } \\
\text { Região ou } \\
\text { área }\end{array}$ & $\begin{array}{l}\text { Coordenada } \\
\text { geográfica }\end{array}$ & $\begin{array}{c}\text { Produção } \\
\text { serapilheira } \\
\left(\mathrm{Mgha}^{-1} \mathrm{ano}^{-1}\right)\end{array}$ & $\begin{array}{c}\text { Ano da } \\
\text { pesquisa }\end{array}$ & Referência \\
\hline Sinop & $\begin{array}{c}\text { Floresta de } \\
\text { Transição } \\
\text { Amazônia- } \\
\text { Cerrado/ } \\
\text { Fazenda } \\
\text { Maracai }\end{array}$ & $\begin{array}{l}11244^{\prime} 43,4^{\prime \prime} \mathrm{S}: \\
55019^{\prime} 25,7^{\prime \prime} \mathrm{O}\end{array}$ & 8 a 10,5 & $\begin{array}{c}2001 a \\
2007\end{array}$ & $\begin{array}{c}\text { Sanches et } \\
\text { al. (2008) }\end{array}$ \\
\hline Sinop & $\begin{array}{c}\text { Floresta de } \\
\text { Transição } \\
\text { Amazônia- } \\
\text { Cerrado / } \\
\text { Fazenda } \\
\text { Maracai }\end{array}$ & $\begin{array}{l}11^{\circ} 24,75^{\prime} \mathrm{S}: \\
55^{\circ} 19,50^{\prime} \mathrm{O}\end{array}$ & 6,57 & $\begin{array}{c}2002 a \\
2003\end{array}$ & $\begin{array}{c}\text { Silva et al. } \\
(2007)\end{array}$ \\
\hline
\end{tabular}

Continua... 


\begin{tabular}{|c|c|c|c|c|c|}
\hline Sinop & $\begin{array}{l}\text { Floresta de } \\
\text { Transição } \\
\text { Amazônia- } \\
\text { Cerrado / } \\
\text { Fazenda } \\
\text { Maracai }\end{array}$ & $\begin{array}{l}11^{\circ} 24.75^{\prime} \mathrm{S}: \\
55^{\circ} 19.50^{\prime} \mathrm{O}\end{array}$ & 7,99 & $\begin{array}{c}2003 a \\
2004\end{array}$ & $\begin{array}{l}\text { Valentini } \\
\text { (2004) }\end{array}$ \\
\hline $\begin{array}{l}\text { Campo Ver- } \\
\text { de }\end{array}$ & $\begin{array}{c}\text { Floresta } \\
\text { Nativa/ } \\
\text { Fazenda São } \\
\text { José da Serra }\end{array}$ & $\begin{array}{l}15^{\circ} 48^{\prime} 00^{\prime \prime} \mathrm{S}: \\
55^{\circ} 26^{\prime} 00^{\prime \prime} \mathrm{O}\end{array}$ & 9,04 & $\begin{array}{c}2003 a \\
2004\end{array}$ & $\begin{array}{c}\text { Fernandes \& } \\
\text { Scaramuzza } \\
(2007)\end{array}$ \\
\hline Juruena & $\begin{array}{c}\text { Floresta } \\
\text { nativa }\end{array}$ & $\begin{array}{l}\text { 10॰25'S: } \\
58 \circ 46^{\prime} \mathrm{O}\end{array}$ & 11,8 & $\begin{array}{c}2003 a \\
2004\end{array}$ & \begin{tabular}{|c} 
Selva et al. \\
$(2007)$
\end{tabular} \\
\hline Sinop & $\begin{array}{l}\text { Floresta de } \\
\text { Transição } \\
\text { Amazônia- } \\
\text { Cerrado / } \\
\text { Fazenda } \\
\text { Maracai }\end{array}$ & $\begin{array}{l}11^{\circ} 24.75^{\prime} \mathrm{S}: \\
55^{\circ} 19.50^{\prime} \mathrm{O}\end{array}$ & 8,2 & $\begin{array}{c}2003 a \\
2004\end{array}$ & $\begin{array}{c}\text { Almeida } \\
\text { (2005) }\end{array}$ \\
\hline $\begin{array}{l}\text { Campo Ver- } \\
\text { de }\end{array}$ & $\begin{array}{c}\text { Floresta } \\
\text { Nativa / } \\
\text { Fazenda São } \\
\text { José da Serra }\end{array}$ & $\begin{array}{l}15^{\circ} 48^{\prime} 00^{\prime \prime} \mathrm{S}: \\
55^{\circ} 26^{\prime} 00^{\prime \prime} \mathrm{O}\end{array}$ & 16,7 & $\begin{array}{c}2004 a \\
2005\end{array}$ & $\begin{array}{l}\text { Gomes } \\
(2006)\end{array}$ \\
\hline Sinop & $\begin{array}{l}\text { Floresta de } \\
\text { Transição } \\
\text { Amazônia- } \\
\text { Cerrado / } \\
\text { Fazenda } \\
\text { Maracai }\end{array}$ & $\begin{array}{l}11^{\circ} 24^{\prime} 43,4^{\prime \prime} \mathrm{S}: \\
55019^{\prime} 25,7^{\prime \prime} \mathrm{O}\end{array}$ & 8,99 & $\begin{array}{c}2004 a \\
2005\end{array}$ & $\begin{array}{l}\text { Silva et al. } \\
\text { (2009) }\end{array}$ \\
\hline Sinop & $\begin{array}{l}\text { Floresta de } \\
\text { Transição } \\
\text { Amazônia- } \\
\text { Cerrado / } \\
\text { Fazenda } \\
\text { Maracai }\end{array}$ & $\begin{array}{l}\text { 1124'43,4"S: } \\
55019^{\prime} 25,7^{\prime \prime} \mathrm{O}\end{array}$ & 11,23 & $\begin{array}{c}2005 a \\
2006\end{array}$ & $\begin{array}{l}\text { Bambi } \\
(2007)\end{array}$ \\
\hline Claudia & $\begin{array}{l}\text { Floresta } \\
\text { decídua } \\
\text { Transição } \\
\text { Amazônia- } \\
\text { Cerrado / } \\
\text { Fazenda } \\
\text { Continental }\end{array}$ & $\begin{array}{l}11^{\circ} 34^{\prime} \mathrm{S}: \\
55^{\circ} 17^{\prime} \mathrm{O}\end{array}$ & 10,6 & $\begin{array}{c}2012 a \\
2013\end{array}$ & $\begin{array}{l}\text { Almeida et } \\
\text { al. (2015) }\end{array}$ \\
\hline
\end{tabular}

Continua... 


\begin{tabular}{|c|c|c|c|c|c|}
\hline Claudia & $\begin{array}{l}\text { Floresta } \\
\text { decídua } \\
\text { Transição } \\
\text { Amazônia- } \\
\text { Cerrado / } \\
\text { Fazenda } \\
\text { Continental }\end{array}$ & $11^{\circ} 35^{\prime} \mathrm{S}, 55^{\circ} 17^{\prime} \mathrm{O}$ & 8,59 & $\begin{array}{c}2012 a \\
2013\end{array}$ & $\begin{array}{l}\text { Almeida et } \\
\text { al. (2015) }\end{array}$ \\
\hline Claudia & $\begin{array}{l}\text { Floresta } \\
\text { decídua } \\
\text { Transição } \\
\text { Amazônia- } \\
\text { Cerrado / } \\
\text { Fazenda } \\
\text { Iracema }\end{array}$ & $\begin{array}{l}11^{\circ} 39^{\prime} \mathrm{S}: \\
55^{\circ} 04^{\prime} \mathrm{O}\end{array}$ & 10,5 & $\begin{array}{c}2012 a \\
2013\end{array}$ & $\begin{array}{l}\text { Almeida et } \\
\text { al. (2015) }\end{array}$ \\
\hline Cotriguaçu & $\begin{array}{l}\text { Floresta } \\
\text { ombrófila } \\
\text { aberta } \\
\text { intacta/ } \\
\text { Fazenda São } \\
\text { Nicolau }\end{array}$ & $\begin{array}{l}09^{\circ} 52^{\prime} \mathrm{S}: \\
58^{\circ} 13^{\prime} \mathrm{O}\end{array}$ & 13,2 & $\begin{array}{c}2012 a \\
2013\end{array}$ & $\begin{array}{l}\text { Almeida et } \\
\text { al. (2015) }\end{array}$ \\
\hline Novo Mundo & $\begin{array}{l}\text { Floresta de } \\
\text { transição } \\
\text { entre } \\
\text { Ombrófila } \\
\text { e Estacional } \\
\text { intacta / } \\
\text { Parque } \\
\text { Estadual do } \\
\text { Cristalino }\end{array}$ & $9^{\circ} 28^{\prime} \mathrm{S}: 55^{\circ} 50^{\prime} \mathrm{O}$ & 10,2 & $\begin{array}{c}2012 a \\
2013\end{array}$ & $\begin{array}{l}\text { Almeida et } \\
\text { al. (2015) }\end{array}$ \\
\hline $\begin{array}{l}\text { Nova Xavan- } \\
\text { tina }\end{array}$ & $\begin{array}{l}\text { Floresta de } \\
\text { Transição } \\
\text { Amazônia- } \\
\text { Cerrado/ } \\
\text { Parque } \\
\text { Municipal } \\
\text { Bacaba }\end{array}$ & $\begin{array}{l}14^{\circ} 42^{\prime} 02,3^{\prime \prime} \mathrm{S}: \\
52^{\circ} 21^{\prime} 02,6^{\prime \prime} \mathrm{O}\end{array}$ & 8,72 & $\begin{array}{c}2011 a \\
2013\end{array}$ & $\begin{array}{l}\text { Valadão et } \\
\text { al. (2016) }\end{array}$ \\
\hline Sinop & $\begin{array}{l}\text { Floresta de } \\
\text { Transição } \\
\text { Amazônia- } \\
\text { Cerrado / } \\
\text { Fazenda } \\
\text { Timboense }\end{array}$ & $\begin{array}{l}\text { 1130'1"S: } \\
55^{\circ} 33^{\prime} 22^{\prime \prime} \mathrm{O}\end{array}$ & 14,5 & $\begin{array}{c}2014 a \\
2015\end{array}$ & $\begin{array}{l}\text { Garrido } \\
\text { (2016) }\end{array}$ \\
\hline
\end{tabular}

TABELA 2 - Produção de serrapilheira na Floresta Amazônica de Mato Grosso 
Na Tabela abaixo são apresentados os dados obtidos no Pantanal de Mato Grosso.

\begin{tabular}{|c|c|c|c|c|c|}
\hline Município & $\begin{array}{l}\text { Tipologia/Re- } \\
\text { gião ou área }\end{array}$ & $\begin{array}{l}\text { Coordenada } \\
\text { geográfica }\end{array}$ & $\begin{array}{c}\text { Produção } \\
\text { serapilheira } \\
\left(\mathrm{Mgha}^{-1} \mathrm{ano}^{-1}\right)\end{array}$ & $\begin{array}{c}\text { Ano da } \\
\text { pesquisa }\end{array}$ & Referência \\
\hline Poconé & 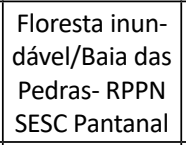 & $\begin{array}{l}16^{\circ} 30^{\prime} 31.139^{\prime \prime} \mathrm{S}: \\
56^{\circ} 24^{\prime} 24.505^{\prime \prime} \mathrm{O}\end{array}$ & 0,629 & $\begin{array}{c}2014 a \\
2015\end{array}$ & $\begin{array}{c}\text { Carneiro } \\
\text { (2015) }\end{array}$ \\
\hline Poconé & \begin{tabular}{|c|} 
Floresta não \\
inundável/Baia \\
das Pedras- \\
RPPN SESC \\
Pantanal \\
\end{tabular} & $\begin{array}{l}16^{\circ} 30^{\prime} 15.202^{\prime \prime} \mathrm{S}: \\
56^{\circ} 24^{\prime} 15.304^{\prime \prime} \mathrm{O}\end{array}$ & 0,435 & $\begin{array}{c}2014 a \\
2015\end{array}$ & $\begin{array}{c}\text { Carneiro } \\
\text { (2015) }\end{array}$ \\
\hline Poconé & $\begin{array}{c}\text { Cambarazal/ } \\
\text { RPPN SESC } \\
\text { Pantanal }\end{array}$ & $\begin{array}{l}16^{\circ} 29^{\prime} 10^{\prime \prime} \mathrm{S}: \\
56^{\circ} 25^{\prime} 26^{\prime \prime} \mathrm{O}\end{array}$ & 10,46 & 2014 & $\begin{array}{c}\text { Dias } \\
\text { (2017) }\end{array}$ \\
\hline Poconé & $\begin{array}{l}\text { Acurizal/ RPPN } \\
\text { SESC Pantanal }\end{array}$ & $\begin{array}{l}16^{\circ} 30^{\prime} 14^{\prime \prime} \mathrm{S}: \\
56^{\circ} 24^{\prime} 17^{\prime \prime} \mathrm{O}\end{array}$ & 7,29 & 2014 & $\begin{array}{c}\text { Dias } \\
(2017) \\
\end{array}$ \\
\hline
\end{tabular}

TABELA 3- Produção de serrapilheira no Pantanal de Mato Grosso

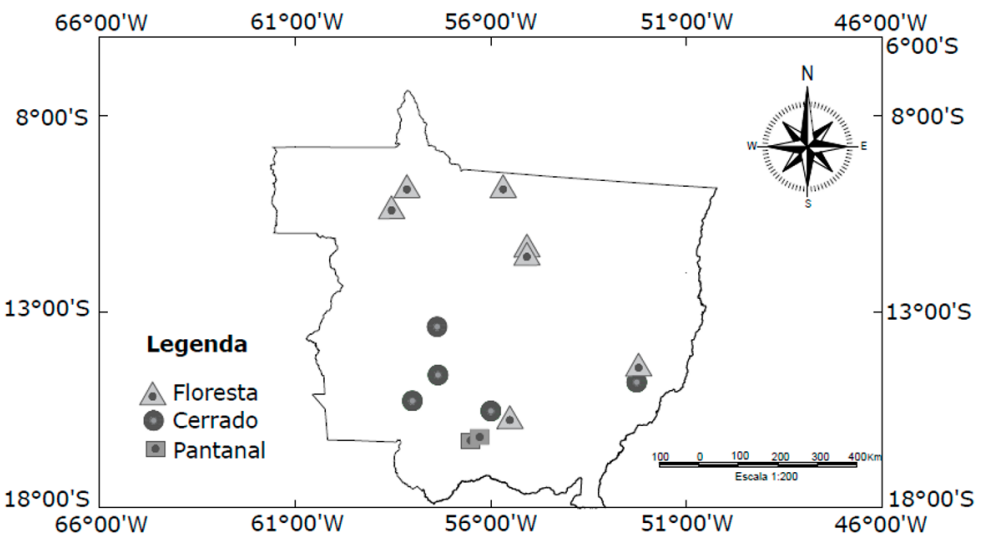

Figura 2. Localização dos pontos de estudos de serapilheira no Estado de Mato Grosso

Antes de realizar a discussão dos resultados apresentados de cada bioma e seus ecótonos, é necessário tecer algumas considerações. Ao analisar os valores de aporte de liteira apresentados nas Tabelas 1, 2 e 3, observam-se algumas disparidades entre os dados no mesmo bioma ou na mesma área de transição. Há inúmeras ra- 
zões para que os valores se apresentem discrepantes. Dentre elas, estão as diferentes épocas de coletas, as metodologias aplicadas, a fragmentação dos biomas, os efeitos de bordas não analisados e as distinções que existem entre as fitofisionomias apresentadas dentro de um mesmo bioma.

Outra observação importante é que os estudos se deram em fragmentos isolados em fazendas ou dentro de parques municipais e estaduais. Justifica-se a escolha dos locais pelo fato de o bioma e suas transformações poderem ser monitorados ao longo do tempo, mas também se confirma, assim, que os biomas se encontram fragmentados e que se conservam apenas em áreas protegidas.

Portanto, a tentativa de comparar os dados aqui apresentados é uma oportunidade de discutir as dificuldades tão inerentes aos trabalhos de campo e como os resultados podem nos indicar as transformações nesses biomas por meio da produção de serapiIheira.

\section{Produção de serrapilheira no Cerrado mato-grossense}

A principal característica fisionômica e estrutural do Cerrado é a coexistência de dois tipos de forma de vida contrastantes: lenhosas (árvores e arbustos) e herbáceas (particularmente gramíneas), cujas proporções variam em resposta a dois principais determinantes, que são disponibilidade de água e fogo (KLINK \& MACHADO, 2005).

Ratter et al. (1997) já apontavam que as áreas de destruição no Cerrado eram muito maiores que na Floresta Amazônica, devido aos avanços da monocultura e ao fogo. Desta forma, não surpreende o fato de o Cerrado ter sido incluído como um dos hotspots para a conservação da biodiversidade mundial (KLINK \& MACHADO, 2005) por sua alta biodiversidade e grau de endemismo, mas também por seu baixo nível de proteção (FIEDLER et al., 2006).

Machado et al. (2004) estimaram que, se mantidas as taxas de desmatamento relatadas $(1,1 \%$ ou 2,2 milhões de hectares de perda anual), o Cerrado deve desaparecer por volta de 2030, visto que atualmente ele mantém apenas $20 \%$ da sua cobertura original. O Cerradão, por exemplo, rara fitofisionomia florestal que 
ocupava originalmente cerca de $1 \%$ da área total do Cerrado, é considerado o mais ameaçado e fragilizado frente às fragmentações e perturbações no bioma (EITEN, 1979; RIBEIRO e WALTER, 2008; SOLÓRZANO et al., 2012).

Solórzano et al. (2012) ressaltaram que no Cerradão de Mato Grosso há maior proporção de espécies florestais, evidenciando a influência da proximidade geográfica com a Amazônia. Assim sendo, há menor produção de serrapilheira no Cerrado sensu stricto quando comparada ao Cerradão (Tabela 1). Alho (1992) atribuiu a diferença ao fato de o Cerrado sensu stricto ser de vegetação semiaberta com árvores espaçadas e pequenas, além de ter pouca biomassa por unidade de área em relação à floresta.

Porém, nos estudos citados em Cerrado Típico, um dos subtipos do Cerrado stricto sensu (BASTOS \& FERREIRA, 2010), verifica-se que os valores de deposição de serapilheira são maiores. No Cerrado Típico, as árvores e arbustos geralmente não ultrapassam a altura de 4 metros, possuem troncos e galhos retorcidos, e a distribuição das espécies no espaço é bastante regular (WALTER, 2006).

O valor médio encontrado por Valadão et al. (2016) na pesquisa em Cerrado Típico (4,06 Mgha $^{-1} \mathrm{ano}^{-1}$ ), em Nova Xavantina, foi quase sete vezes e meia maior que o encontrado por Silva et al.(2007) em Cerrado stricto sensu $\left(0,62 \mathrm{Mgha}^{-1} \mathrm{ano}^{-1}\right)$. É importante ressaltar que as duas pesquisas foram realizadas no mesmo Parque Municipal Bacaba e, pelas coordenadas citadas nos trabalhos, são formações savânicas vizinhas. Uma das possíveis causas da discrepância de valores pode ser o intervalo de tempo de uma década entre a realização delas. A diferença de idade entre os povoamentos tem uma influência direta na deposição, devido aos distintos estágios de desenvolvimento das plantas. Butler et al. (2012) consideraram o índice de área foliar IAF de cada local de estudo um fator importante para ajudar a elucidar a diferença nos valores de deposição de liteira no Cerrado stricto sensu. Outra observação importante é que há um aumento da deposição da serapilheira até o ponto em que as árvores atingem a maturidade. Depois, há um ligeiro decréscimo ou até mesmo a estabilização das taxas de deposição (VITAL et al., 2004).

Valores maiores de Cerrado Típico também foram encontrados em estudos em dois parques urbanos de Cuiabá. O valor de 6,9 
Mgha $^{-1} \mathrm{ano}^{-1}$, encontrado em área do Parque Mãe Bonifácia por Pereira (2017), servirá como norte para os próximos trabalhos no local. No estudo utilizou-se apenas um coletor de serapilheira e limitaram-se, assim, a dimensão e o gradiente da liteira. A produção anual de biomassa registrada por Valentini et al. (2014) em um fragmento revegetado de Cerrado no Parque Estadual Massairo Okamura (11,89 $\left.\mathrm{Mgha}^{-1} \mathrm{ano}^{-1}\right)$ é superior aos registros em outros estudos de fragmentos de Cerrado de Mato Grosso. O valor encontrado deve-se ao fato de que a maioria das espécies usadas na revegetação da área apresentou distribuição para mais de um domínio fitogeográfico. Somado a isso, apesar de estarem em um estádio avançado de sucessão, as espécies não haviam atingido ainda seu estado de equilíbrio dinâmico. $O$ grande número de espécies zoocóricas e não pioneiras e o fato de a altura das espécies não seguir padrão unimodal também corroboram o resultado (MORAIS et al., 2014).

\section{Produção de serrapilheira nas Florestas mato-grossenses}

Aproximadamente $54 \%$ da área do Estado de Mato Grosso é ocupada pelo bioma Amazônico. Mas, em função da ocorrência de três biomas bastante distintos no Estado, um total de 43,7\% é caracterizado como área de transição entre biomas. $\mathrm{O}$ avanço da pecuária e cultivo da soja em direção ao norte do Estado de Mato Grosso representa uma forte ameaça à biodiversidade amazônica, principalmente nas áreas de transição da Amazônia para o Cerrado. Aárea de maior pressão antrópica na região tem sido o denominado arco do desflorestamento (ou "arco do desmatamento" ou "arco do povoamento"), que corresponde ao leste do Pará, oeste do Maranhão, norte do Mato Grosso e Rondônia, onde a concentração humana é mais elevada (OLIVEIRA \& SANTOS, 2010).

Além da perda do carbono para a atmosfera, o desmatamento também está relacionado à redução do regime de chuvas. O clima sofre uma alteração com a derrubada de grandes áreas com matas, causando períodos mais extensos de estiagem e diminuição na umidade relativa do ar, visto que a transpiração das folhas é um dos fatores fundamentais de regulação da umidade do ar e da temperatura nos ambientes (SILVA \& LOPES, 2016). 
Em função da pressão sobre a Floresta e sua consequente fragmentação, ao analisar os dados da Tabela 2, é importante perceber que a maioria dos autores fez seus estudos em um transecto dentro de um fragmento do bioma. Nos trabalhos não houve relato dos efeitos de borda, que pode ser entendido como o resultado da interação entre dois ecossistemas adjacentes, separados por transição abrupta (MURCIA, 1995). A abordagem que considera a influência da estrutura da paisagem em padrões de produção de serapilheira é ainda muito pouco explorada no Brasil (VIDAL et al., 2007). Assim, tem-se ainda uma visão limitada sobre a capacidade dos fragmentos de manter os processos ecológicos, pois a distância que as alterações/impactos avançam desde a borda para o interior dos fragmentos não foi medida (CORREA LAGOS, 2017).

Brasil et al. (2013) foram os únicos a considerar o efeito de borda na produção de serapilheira em um remanescente de Cerradão (Transição Amazônia-Cerrado) no Parque Municipal Bacaba, em Nova Xavantina. É nítida a diminuição de 31,8\% no aporte de biomassa na borda $\left(7,65 \mathrm{Mgha}^{-1}\right)$ em relação ao interior do fragmento (11,2 $\left.\mathrm{Mgha}^{-1}\right)$. É importante ressaltar que o objetivo principal do estudo foi, segundo os autores, realizar um levantamento rápido e exploratório, a fim de comparar diferentes partes de uma mesma área (gradiente borda-centro) e em uma mesma época do ano. Desta forma, foi usado outro tipo de coletor, o Marinom-Hay (MARIMON JÚNIOR \& HAY, 2008), que mede a biomassa de uma camada de serapilheira. É uma estimativa menos precisa quando comparada aos modelos de coletores de serapilheira apresentados na metodologia desse capítulo, mas que demanda menos tempo e recursos:

Na Tabela 2 são citados seis trabalhos realizados na Fazenda Maracaí, em uma área de Floresta de transição Cerrado-Floresta Amazônia. Ela se encontra em Sinop, norte do Estado de Mato Grosso. O trabalho de Sanches et al. (2008) originou-se de uma compilação de dados de 2001 a 2007 pelo grupo de Pesquisa da Pós-graduação em Física Ambiental da UFMT. Observa-se, nessas pesquisas, que houve uma variação de 6,5 a 11,23 $\mathrm{Mgha}^{-1} \mathrm{ano}^{-1}$ na produção de serapilheira do fragmento. Observou-se também que ao longo dos sete anos houve aumento no aporte de biomassa. 
Poggiani \& Schumacher (2000) explicaram que é crescente a deposição de serapilheira até as árvores alcançarem idade de maturidade, quando há o fechamento das copas.

Almeida et al. (2015) fizeram seus trabalhos em Cláudia, na Fazenda Continental, área muito próxima à Fazenda Maracaí. Apesar de também terem analisado a deposição de serapilheira na Floresta de transição Cerrado-Floresta Amazônia, a característica principal do estudo, realizado entre 2012 e 2013, foi que as áreas avaliadas com produção de 10,6 $\mathrm{Mgha}^{-1} \mathrm{ano}^{-1}$; 8,59 Mgha${ }^{-1}$ ano $^{-1}$ e 10,5 Mgha-1 $^{-1}$ nno $^{-1}$ tinham sofrido exploração de madeira há 12,19 e 33 anos, respectivamente. Os valores encontrados estão dentro da faixa dos estudos citados anteriormente da Fazenda Maracaí. Porém, só seria possível uma comparação mais fidedigna entre as áreas da Fazendo Maracaí e Continental caso as pesquisas tivessem sido realizadas na mesma época. Almeida et al. (2015) destacaram o fato de que, durante a exploração madeireira, ocorreu uma diminuição na área basal da vegetação. A medida que as áreas foram contidas dessa exploração, houve um aumento gradual na área basal da vegetação, começando pelas espécies pioneiras, até a substituição dessas por espécies secundárias e de clímax (SILVA et al., 1995). Como as espécies pioneiras, nos primeiros anos de vida, investem mais recursos na produção de folhas do que as espécies clímax, foi observado no trabalho de Almeida et al. (2015), maior deposição de liteira nesse estádio sucessional do que nos posteriores (BAZZAZ \& PICKETT, 1980).

O trabalho de Garrido (2016) foi realizado de 2014 a 2015 na Fazenda Timboense, vizinha às Fazendas Maracaí e Continental. Ao observar a deposição de biomassa de $14,5 \mathrm{Mgha}^{-1} \mathrm{ano}^{-1}$ no estudo, podem ser pensadas algumas hipóteses. Ele poderia ser o valor de clímax, mas seriam necessárias coletas posteriores para chegar a essa conclusão. Outra possibilidade seria uma tentativa da floresta em produzir mais folhedo para garantir sua estabilidade no local. Segundo Vitousek \& Sanford Jr. (1986), a manutenção do equilíbrio nutricional das florestas tropicais em solos distróficos depende da contínua produção e decomposição da camada de serapilheira do solo florestal, processo que garante a constante reposição de nutrientes à vegetação. 
A deposição de necromassa em Florestas Nativas de Juruena, com 11,8 $\mathrm{Mgha}^{-1} \mathrm{ano}^{-1}$ (Selva et al., 2007); Campo Verde, 9,04 Mgha $^{-1}$ ano $^{-1}$ (Fernandes \& Scaramuzza, 2007); Cotriguaçu, 13,2 Mgha $^{-1}$ ano $^{-1}$ (Almeida et al., 2015); e Novo Mundo, 10,2 Mgha${ }^{-1} a_{n o}{ }^{-1}$ (Almeida et al., 2015) mostram valores próximos aos demais estudos. Entretanto, é muito difícil compará-los tanto por terem sido realizados em épocas diferentes quanto pela diversidade fitofisiônomica existente nas florestas de Mato Grosso.

Um valor que se destaca foi o do trabalho de Gomes (2006) na Floresta Nativa da Fazenda São José da Serra, em Campo Verde, que entre 2004 e 2005 encontrou uma deposição de liteira igual a $16,7 \mathrm{Mgha}^{-1} \mathrm{ano}^{-1}$. No mesmo local, o trabalho de Fernandes \& Scaramuzza (2007), um ano antes, encontrou um valor de 9,04 Mgha $^{-1} \mathrm{ano}^{-1}$. Como usaram a mesma metodologia, o valor muito superior em um prazo de um ano pode estar associado a eventos que ocorreram vizinhos ao fragmento, mas que não foram relatados pelo autor.

\section{Produção de serrapilheira no Pantanal mato-grossense}

No Pantanal são características as diversas unidades de vegetação, formando um grande mosaico, com frequentes mudanças abruptas. Porém, o aspecto que mais se destaca no bioma é a combinação de vegetação mésica e xérica, crescendo lado a lado. As razões para as manchas na paisagem com diferentes comunidades são as flutuações anuais do nível de água, que inundam sazonalmente essa planície. Assim, sua topografia e sazonalidade climática, aliadas ao vai e vem das águas, aperfeiçoam as linhagens florísticas e as espécies. Elas se tornam adaptadas a diferentes condições, o que resulta na fisionomia característica dos diversos pantanais (PRANCE \& SCHALLER, 1982; FORMAN \& GODRON, 1986; NEIFF, 1990, 2001; CUNHA et al. 2007; FANTIN-CRUZ et al., 2010).

Variando de um tipo para outro em pequenas distâncias, é frequente ocorrer cerradão, floresta estacional e mata ciliar em apenas 100 metros de cordilheira ou capão (POTT \& POTT, 2003). É preciso considerar que, além dos ciclos anuais de inundação e rebaixamento das águas, a aglutinação em períodos de vários anos 
com inundações mais volumosas, alternadas com períodos mais secos, determina essa forte zonação na distribuição da vegetação, principalmente a vegetação rasteira (VALLS et al., 2003).

De acordo com Pott \& Pott (1997), o ecossistema pantaneiro pode ser dividido em até 10 sub-regiões diferentes. Cada fisionomia resulta de uma interação única de fatores edáficos, hidrológicos e biogeográficos. As sub-regiões são: Cáceres, Poconé, Barão de Melgaço, Paraguai, Paiaguás, Nhecolândia, Abobral, Aquidauana, Miranda e Nabileque.

No estado de Mato Grosso, conhecido como Pantanal Norte, estão as seguintes sub-regiões: Poconé, Cáceres e Barão do Melgaço. O local dos estudos citados na Tabela 3 é a sub-região de Barão de Melgaço. Ela se encontra entre os paralelos 16 a 17ㅇ S e meridianos 56ㅇ a 57ㅇ W, onde está situada a Reserva Particular do Patrimônio Natural do Serviço Social do Comércio (RPPN SESC Pantanal), entre os Rios Cuiabá e São Lourenço (BEIRIGO et al., 2011).

São reconhecidas na RPPN SESC Pantanal sete fisionomias vegetais: Cerrado stricto sensu, Cerradão, Cambarazal, Campo com Murunduns, Floresta Estacional com Acuri, Campo e outras fisionomias e ecótonos (HASENACK et al., 2003). O local é caracterizado por períodos de inundação entre dezembro e maio e de seca entre junho e novembro, com a estação chuvosa se estendendo de outubro a abril (SIGNOR et al., 2010).

Devido a sua posição fitogeográfica, que o coloca em contato com diferentes tipos de vegetação circundantes, o Pantanal se torna vulnerável a invasão de plantas daninhas (invasoras). Uma espécie bastante característica da região onde ocorreram os estudos (Tabela 3) é a Vochysia divergens Pohl, formação monodominante conhecida como Cambarazal. Como pioneira, vem invadindo a área de campo desde o ciclo hiper-hídrico de 1974, que se estendeu até meados da década de 1990 (SANTOS et al., 2006).

Em uma área com dominância de Scheelea phalerata (Mart. ex Spreng.) Burret, conhecida como Acurizal, e em menor grau de dominância de Combretum leprosum Mart. e Anadenanthera falcata (Benth.) Speg, respectivamente conhecidas como carne-de-vaca e angico do cerrado (SANCHES et al., 2015), a produção 
de serrapilheira foi menor quando comparada com a floresta de Cambarazal (DIAS, 2017).

Além da topografia, que determina a inundação que ocorre no Pantanal, a composição da vegetação é um fator importante que pode explicar as diferenças entre as florestas quanto à produção de serrapilheira (MALHI et al., 2009). Tais distinções também podem ser atribuídas às diferenças de idade da vegetação arbórea, à diversidade (riqueza e abundância) de espécies (VOURLITIS et al., 2012), à quantidade de material vegetal que cai da parte aérea de cada floresta e à taxa de decomposição da serrapilheira (VALENTINI et al., 2008).

O efeito das condições de seca sobre a vegetação e, por conseguinte, o aumento da produção de serrapilheira, ocorre em florestas com característica decídua e semidecídua, perenes, em áreas baixas e ao longo dos cursos de água no Pantanal (RATTER et al., 1978; JUNK et al., 2006; SILVA, 2013; DIAS, 2017). Além das condições climáticas, o nível de complexidade de reações da biota, devido à sazonalidade em área úmida, resulta na existência de espécies singulares (JUNK et al., 2006). Elas possuem diferentes respostas no seu comportamento vegetacional graças às alterações constantes no sistema solo-planta-atmosfera.

Conforme apresentado na Tabela 3, a produção de serrapiIheira no Cambarazal e no Acurizal (DIAS, 2017) possui distinções, que podem ser explicadas pelas diferenças das alturas das lâminas d'água e pelo tempo de duração da inundação nas florestas. Ambos os fatores ocorrem devido à topografia das áreas e aos tipos de solos (ZEILHOFER \& SCHESSI, 2000). É importante ressaltar que a diferença de altitude entre as duas áreas do Cambarazal e do Acurizal é de, aproximadamente, dois metros. Também se deve considerar que as florestas mais densas do Pantanal apresentam umidade relativa do ar maior que 70\% (HASENACK et al., 2003), superior às áreas menos densas. A umidade influencia indiretamente na produção de serapilheira, pois está associada ao balanço hídrico local.

Comumente, a produção de serrapilheira em florestas sazonalmente inundáveis é maior se comparada às florestas adjacentes em áreas mais elevadas. É um indicativo de que a produção da floresta inundada é, em geral, maior que das florestas de terras 
altas no mesmo local (HAASE, 1999). Entretanto, observou-se que mesmo que se trate de uma floresta com a mesma dominância, pode ocorrer discrepância na produção de serrapilheira, devido às condições locais. Por exemplo, Dias (2017) obteve maiores valores de produção de serapilheira no Acurizal que Carneiro (2015), estudo realizado na mesma localidade. Um dos fatores que explica a diferença é a topografia das áreas dos estudos.

Além da topografia e da formação vegetal, a duração e a lâmina d'água de inundação também influenciam tanto no aporte de biomassa como na sua taxa de decomposição, ou seja, na velocidade de decomposição da serrapilheira (LIU et al., 2010). Logo, ela está presente nos processos de ciclagem de nutrientes e no desenvolvimento da vegetação. Quando se trata do Pantanal, uma área inundável, deve-se considerar que ele recebe um aporte de sedimentos oriundos da bacia hidrográfica a montante, que também terão interação com o material decíduo. A discussão sobre os fatores é complexa, pois além deles - topografia, tempo de inundação e formação vegetal -, deve-se destacar a influência do $\mathrm{pH}$ do solo, da densidade da população de organismos decompositores e das condições ambientais do local, como precipitação, umidade e temperatura do ar, umidade do solo, entres outros (CALDEIRA et al., 2008).

Correa Lagos (2017) realizou uma abordagem importante sobre a produção de serapilheira para o bioma Cerrado, revisando trabalhos no Cerrado brasileiro de 1970 a 2015. Apontou dificuldades em comparar os dados dessas publicações devido às lacunas de dados e vieses nem sempre especificados. A seu exemplo, o presente trabalho não se mostrou diferente. Tal como a autora, observa-se que a falta de padronização na metodologia, aliada às diferentes fitofisionomias dos biomas e desconhecimento do seu entorno, impossibilita sistematizar os dados e proporcionar a disseminação (ou extrapolação) sobre a produção de serrapilheira para cada bioma de Mato Grosso. Caminhos para uma possível recuperação de áreas antropizadas são encontrado em recortes literários para os diferentes biomas. 


\section{CONSIDERAÇÕES FINAIS}

Estudos em regiões com altos índices de desmatamento devem ser priorizados para entender quais são os efeitos dessas alterações em curto e longo prazo sobre a produção de serrapilheira. Tais estudos devem ser contínuos, com metodologia padronizada, incluindo os efeitos de borda e conhecimento das alterações ocorridas nas áreas vizinhas.

A revegetação com espécies do bioma é uma alternativa para áreas modificadas ou degradadas, visto que a produção de liteira em Valentini et al. (2014), estudo realizado no Cerrado, foi superior a outras áreas do bioma.

A descontinuidade do desmatamento no estudo de Almeida et al. (2015) também se apresentou eficaz em áreas de floresta, mostrando que com o tempo houve uma produção de serapilheira próxima a outras áreas de florestas intactas.

Como o estudo de serrapilheira no Pantanal Norte é recente, carece de mais dados que apontem como se comporta a deposição do material decíduo, em especial para áreas com espécies invasoras.

\section{REFERÊNCIAS}

ABREU, J. R.S.P. Dinâmica da serrapilheira em um trecho de floresta atlântica secundária em área urbana do Rio de Janeiro. 2006. 79 p. Dissertação (Mestrado em Ciências) - Ciências Ambientais e Florestais, Universidade Federal Rural do Rio de Janeiro. 2006.

AERTS, R. Climate, leaf litter chemistry, and leaf litter decomposition interrestrial ecosystems: A triangular relationship, Oikos, v. 79, p.439-449, 1997.

AGUIAR, L. M. S.; CAMARGO, A. J. A. Cerrado: ecologia e caracterização. Planaltina, DF: Embrapa Informação Tecnológica, 2004. 249p.

ALHO, C.J.R. A teia da vida: uma introdução à ecologia brasileira. Editora Objetiva, Rio de Janeiro. 1992. 160p.

ALMEIDA, E. J.; LUIZÃO, F. RODRIGUES, D. J. Produção de serrapilheira em florestas intactas e exploradas seletivamente no sul da Amazônia em função da área basal da vegetação e da densidade de plantas. Acta Amazônica, v. 45, n.2, p. 157-166, 2015.

ALMEIDA, E.D. 2005. 75p. Retorno de nitrogênio e fósforo em floresta de transição no noroeste de Mato Grosso. Dissertação de Mestrado (Pós-graduação em Física e Meio ambiente) Universidade Federal de Mato Grosso, Cuiabá-MT, 2005. 
BAMBI, P. Variação sazonal do índice da área foliar e sua contribuição na composição da serapilheira e ciclagem de nutrientes na floresta de transição no norte do Mato Grosso. 2007. 99f. Dissertação de Mestrado (Pós-graduação em Física e Meio ambiente) Universidade Federal de Mato Grosso, Cuiabá-MT, 2007.

BASTOS, L. M.; FERREIRA, I. M. Composições fitofisionômicas do bioma Cerrado: Estudo sobre o subsistema de Vereda. Espaço em Revista, v.12, p.99-108, 2010.

BAZZAZ, E. A.; PICKETT, S. T. A. Physiological ecology of tropical succession: A comparative review. Annual Review of Ecology, Evolution, and Systematics, v.11, p.287-310, 1980

BEIRIGO, R.M.; VIDALTORRADO, P.; STAPE, J.L.; COUTO, E.G.; ANDRADE, G.R.P. Solos da Reserva Particular do Patrimônio Natural SESC Pantanal. Rio de Janeiro: SESC, 2011. 75 p.

BRASIL, S. L.; GIEHL, N. F. S.; SANTOS, J. O.; SANTOS, A. O.; MARIMON, B. S.; JUNIOR, B. H. M. Efeito de borda sobre a camada de serrapilheira em área de cerradão no Leste do Mato Grosso. Revista Biotemas, v.34, n. 3, p. 37-47, 2013.

BUTLER, A.; MEIR, P.; SAIZ, G. ; MARACAHIPES, L. ; MARIMON, B. S.; GRACE, J. Annual variation in soil respiration and its component parts in two structurally contrasting woody savannas in Central Brazil. Plant and soil, v. 352, n. 1-2, p. 129-142, 2012.

CALDEIRA, M. V. W; VITORINO, M. D.; SCHAADT, S. S.; MORAES, E.; BALBINOT, R., Quantificação de serapilheira e de nutrientes em uma Floresta Ombrófila Densa. Semina. Ciências Agrárias (online), v.29 p. 53-68, 2008.

CAMARGO, F. F.; SOUZA, R.T.; COSTA, R.B. Etnoecologia e etnobotânica em ambientes de Cerrado no Estado de Mato Grosso. Interações, v. 15, n.2, p. 353-360, 2014.

CARNEIRO, E. M. S. Avaliação da Produção de Serapilheira e da Dinâmica de Macronutrientes em duas Áreas de regimes Hídricos Distintos no Pantanal De Poconé-MT. 2015. 69p. Dissertação de Mestrado (Pós-graduação Ciências Ambientais). Universidade de Cuiabá. Cuiabá-MT. 2015

CLARK, A.D.; BROWN, S.; KICLIGHTER, D.W.; CHAMBERS, J.Q.; THOMLINSON, J. R.; $\mathrm{NI}, \mathrm{J}$; HOLLAND, E. A. Net primary production in tropical forests: an evaluation and synthesis of existing field data. Ecological Applications, v.11, n.2, p.371-384, 2001.

CORREA LAGOS, M. C. Efeito de borda em fragmentos do Cerrado e Mata Atlântica. 2017. 86f. Tese de Doutorado (Pós-graduação Ciências Ambientais). Universidade Federal de Goiás. Goiânia-GO. 2017.

CUNHA, C.N.; JUNK, W.J.; LEITÃO-FILHO, H.F. Woody vegetation in the Pantanal of Mato Grosso, Brasil: a preliminary typology. Amazoniana, v.19, n.3-4, p.159-184, 2007.

DELITTI, W.B.C. Estudos de ciclagem de nutrientes: instrumentos para a análise funcional de ecossistemas terrestres. Oecologia Brasiliensis, v.1, p.469-486, 1995.

DIAS, V. R. M. 2017. 131f. Ciclagem de carbono e nutrientes em florestas sazonalmente inundáveis no Pantanal mato-grossense. Tese de doutorado (Programa 
de Pós-Graduação em Física Ambiental) Universidade Federal de Mato Grosso. Cuiabá-MT, 2017.

DIAS, H. C. T.; OLIVEIRA-FILHO, A. T. Variação temporal e espacial da produção de serapilheira em uma área de floresta estacional semidecídua montana em Lavras-MG. Revista Árvore, v. 21, n. 1, p. 11-26, 1997.

DOMINGUES, M.S.; BERMANN, C. O arco de desflorestamento na Amazônia: da pecuária à soja. Ambiente \& Sociedade, v.15, n.2, p.1-22, 2012.

EITEN, G. Formas fisionômicas do Cerrado. Revista Brasileira de Botânica, v.2, n.2, p.139-148, 1979.

FANTIN-CRUZ, I.; GIRARD, P.; ZEILHOFER, P.; COLLISCHONN, W.; CUNHA, C.N. Unidades fitofisionômicas em mesoescala no Pantanal Norte e suas relações com a geomorfologia. Biota Neotropica, v.10, n.2, p.31-38, 2010.

FERNANDES, F. C. S.; SCARAMUZZA, W. L. M. P. Produção e decomposição da liteira em fragmento florestal em Campo Verde (MT). Revista Ciências Agrárias, n. 47, p. 173-186, 2007.

FIEDLER, N. C.; MERLO, A. M.; MEDEIROS, M. B. Ocorrência de incêndios florestais no Parque Nacional da Chapada dos Veadeiros, Goiás. Ciência Florestal, v. 16, n. 2, p. 153-161, 2006.

FORMAN, R.T.T.; GODRON, M. Landscape Ecology. John Wiley \& Sons, New York, 1986. 619p.

GARRIDO, G. Estudo do aporte de nutrientes via serapilheira em um ecótono de Floresta Tropical e Cerrado na região de Sinop - MT. 2016. 52f. Dissertação de Mestrado (Programa de Pós-graduação em Ciências Ambientais). Universidade de Cuiabá, Cuiabá-MT. 2016.

GOMES, A. P. Produção de serrapilheira por ajuste de equações em floresta nativa e em capoeira. 2006. 46 f. Dissertação de Mestrado (Pós-graduação em Agricultura Tropical). Universidade Federal de Mato Grosso, Cuiabá-MT. 2006.

GRACE, J.; MAHLI, Y.; HIGUCHI, N.; MEIR, P. Productivity of tropical rain forests. In ROY, J., SAUGIER, B.; MOONEY, H. A. (eds.). Terrestrial Global Productivity. Academic Press, Inc., San Diego, CA. 2001. p.401-428.

HAASE, R. Litterfall and nutrientes return in seazonally flooded and non-flooded forest of the Pantanal, Mato Grosso, Brazil. Forest. Ecology and Management, v. 117, p. 129-147, 1999.

HASENACK, H.; CORDEIRO, J. L. P. \& HOFMANN, G. S. O clima da RPPN Sesc Pantanal - Relatório Técnico. Universidade Federal do Rio Grande do Sul, Porto Alegre. 2003. 31p.

INSTITUTO BRASILEIRO DE GEOGRAFIA E ESTATÍSTICA (IBGE). Departamento de Recursos Naturais e Estudos Ambientais. Latitude e longitude do estado de Mato Grosso. Brasil, 2015. 
JUNK, W. J.; BROWN, M.; CAMPBELL, I. C.; FINLAYSON, M.; GOPAL, B.; RAMBERG, L.; WARNER, B.G. The comparative biodiversity of seven globally important wetlands: A synthesis. Aquatic Sciences, v. 68, n.3, p. 400-414, 2006.

KELLER, M.; ALENCAR, A.; ASNER, G. P.; BRASWELL, B.; BUSTAMANTE, M. et al. Ecological research in the large-scale biosphere-atmosphere experiment in Amazonia: Early results. Ecological Applications, v.14, p. S3-S16, 2004.

KLINK, C. A.; MACHADO, R. B. A conservação do cerrado brasileiro. Desafios e oportunidades para a conservação da biodiversidade no Brasil. Megadiversidade, v. 1 , n. 1 , p. 147-155, 2005.

KLUMPP, A. Utilização de bioindicadores de poluição em condições temperadas e tropicais. In: MAIA, N. B.; MARTOS, H. L.; BARRELLA, W. (Eds.). Indicadores ambientais: conceitos e aplicações. São Paulo: EDUC/COMPED/INEP, 2001. p.77-94.

LIU, P.; WANG, Q.; BAI, J.; GAO, H.; HUANG, L.; XIAO, R. Decomposition and return of $\mathrm{C}$ and $\mathrm{N}$ of plant litters of Phragmites australis and Suaeda salsa in typical wetlands of the Yellow River Delta, China. Procedia Environmental Sciences, v. 2, p. 1717-1726, 2010.

MACHADO, R. B., RAMOS NETO, M. B., PEREIRA, E. F., CALDAS, D. A., GONÇALVES, N. S., SANTOS, K. T. e STEININGER, M. Estimativas de perda da área do Cerrado brasileiro. Relatório técnico não publicado. Conservação Internacional, Brasília, DF. 2004.

MAMAN, A. P.; SILVA, C. J.; SGUAREZI, E. M.; BLEICH, M. E. Produção e acúmulo de serapilheira e decomposição foliar em Mata de Galeria e Cerradão no sudoeste de Mato Grosso. Revista de Ciências Agro-Ambientais, v.5, n.1, p.71-84, 2007.

MARIMON - JUNIOR, B. H., HAY, J. D. A new instrument for measurement and collection of quantitative samples of the litter layer in forests. Forest Ecology and Management, v.255, p.2244 - 2250, 2008.

McCANN, K.S. The diversity-stability debate. Nature, v. 405, p.228-233, 2000.

MITCHELL, R.J.; AULD, M.H.D.; Le DUC, M.G.; MARRS, R.H. Ecosystem stability and resilience: a review of their relevance for the conservation management of lowland heaths. Perspectives in Plant Ecology, Evolution and Systematics, v.3, p. 142-160, 2000.

MONTEZUMA R. C. M. Produção e reabilitação funcional do piso florestal em clareira de deslizamento: Parque Nacional da tijuca, Rio de Janeiro. Anuário do Instituto de Geociências 2005; v.28, n.2, p.155-156, 2005.

MORAIS, R. F; VALENTINI, C. M. A.; CORRÊA, B. M. B. Composição florística e características estruturais e ecológicas da vegetação de um fragmento revegetado de Cerrado em área urbana no município de Cuiabá-MT. In: Múltiplos olhares sobre a biodiversidade. Volume III. Pasa, M. C. (org) Paco Editorial, 2014. p. 185-204.

MURCIA, C. Edge effects in fragmented forests: implications for conservation. Trends in Ecology and Evolution, v.10, p.58-62, 1995. 
NEIFF, J.J. Ideas for an ecological interpretation of the Paraná. Interciencia, v. 15, n.6, p. 424-441, 1990.

NEUBURGER, M. Estratégias de sobrevivência entre tradição e inovação - exemplos da produção familiar em Mato Grosso, Brasil. Actas latinoamericanas de Varsovia, v. 26, p. $45-62,2003$.

NUNES, J. R. S.; SILVA, C. J.; FERRAZ, L. Mato Grosso e seus biomas: Biodiversidade, desafios sócio-ambientais, unidades de conservação, iniciativas de políticas públicas e privadas para a conservação. Revista Gestão Universitária, v. 07, p. 01-28, 2017.

OLIVEIRA, A. C. M; SANTOS, P. G. P. Introdução Geral. In: Oliveira, A. C. M; SANTOS, J. B.; SANTOS-COSTA, M. C. (organizadoras). Os animais da Tanguro, Mato Grosso: diversidade na zona de transição entre a Floresta Amazônica e o Cerrado. Belém-PA: MPEG, UFPA, IPAM, 2010. 116p.

PEREIRA, S. P. Dinâmica temporal do efluxo de $\mathrm{CO}_{2}$ em fragmento do cerrado na cidade de Cuiabá-MT. 2017. 44p. Dissertação de Mestrado (Pós-graduação Ciências Ambientais). Universidade de Cuiabá. Cuiabá-MT. 2017.

POGGIANI, F.; SCHUMACHER, M.V. Ciclagem de nutrientes em florestas nativas. In: GONÇALVES, J.L.M.; BENEDETTI, V., ed. Nutrição e fertilização florestal. Piracicaba: IPEF, 2000. cap.10, p.287-308.

POST, W. M.; EMANUEL, W. R.; ZINKE, P. J.; STANGENBERGER, A. G. Soil carbon pools and world life zones. Nature, v. 298, p.156-159, 1982.

POTT, V. J.; POTT, A. Checklist das macrófitas aquáticas do Pantanal, Brasil. Acta Botanica Brasilica, v. 11, n.2, p. 215-227, 1997.

POTT, V.J.; POTT, A. Dinâmica da vegetação aquática do Pantanal. In: THOMAZ S.T. \& BINI, M.B.(eds.) Ecologia e manejo de macrófitas aquáticas. Maringá: EDUEM, p.143-162. 2003.

PRANCE, G.T.; SCHALLER, G.B. Preliminary study of some vegetation types of the Pantanal, Mato Grosso, Brazil. Brittonia, v. 34, n.2, p.228-251, 1982.

RATTER, J. A.; ASKEW, G. P.; MONTGOMERY R. F.; GIFFORD, D. R. Observations on the vegetation of northeastern Mato Grosso. II. Forests and soils of the Rio SuiaMissu area. Proc. Roy. Soc. Lon. Ser. B 203, p. 191-208, 1978.

RATTER, J. A.; RIBEIRO, J. F.; BRIDGEWATER, S. The brazilian cerrado vegetation and threats to its biodiversity. Annals of Botany 80, v.3, p. 223-230, 1997.

RIBEIRO, J.F.; WALTER, B.M.T. As principais fitofisionomias do Bioma Cerrado. In: SANO, S.M.; ALMEIDA, S.P.; RIBEIRO, J. F. eds. Cerrado: ecologia e flora. Embrapa Cerrados, Planaltina. p.151 -212. 2008.

RODRIGUES, E. Efeito de bordas em fragmentos de floresta. Cadernos da Biodiversidade, v. 1, n. 2, p.1-5, 1998. 
SANCHES, L.; DIAS, V. R. M.; SALLO, F. S.; PALÁCIOS, R. S.; VALENTINI, C. M. A.; NOGUEIRA, J. S. Composição florística e estrutura da comunidade vegetal nas fitofisionomias Cambarazal e Acurizal no Norte do Pantanal. In: (Org.) PASA, M. C. Múltiplos Olhares sobre a Biodiversidade IV. Cuiabá: Carlini \& Carniato Editorial, 368p., 2015.

SANCHES, L.; VALENTINI, C.M.A.; PINTO JÚNIOR, O.B.; NOGUEIRA, J.S.; VOURLITIS, G.L.; BIUDES, M.S. et al. Seasonal and interannual litter dynamics of a tropical semideciduous forest of the southern Amazon Basin, Brazil. Journal of Geophysical Research, v.113, p 1-9, 2008.

SANTOS, J.R. Avanços das pesquisas e aplicações de sensoriamento remoto no monitoramento da paisagem: contribuições aos estudos do Pantanal. Anais...In: 1o Simpósio de Geotecnologias no Pantanal, Campo Grande, Brasil, 11-15 novembro 2006, Embrapa Informática Agropecuária/INPE, p.675-683.

SCHLESINGER, W. H. Biogeochemistry: An analysis of global change. Academic Press, Inc., San Diego, CA, USA. 443p. 1991.

SCORIZA, R. N.; PEREIRA, M. G.; PEREIRA, G. H. A.; MACHADO, D. L.; SILVA, E. M. R. Métodos para coleta e análise de serrapilheira aplicados à ciclagem de nutrientes. Floresta e Ambiente, v. 2, n. 2, p. 01-18, 2012.

SELVA, E.C.; COUTO, E.G.; JOHNSON, M.S.; LEHMANN, J. Litterfall production and fluvial export in headwater catchments of the southern Amazon. Journal of Tropical Ecology, v.23, p.329-335, 2007.

SIGNOR, C. A.; FERNANDES, I. M.; PENHA, J. M. F. O Pantanal e o Sistema de Pesquisa. In: FERNANDES, I. (org.). Biodiversidade no Pantanal de Poconé. Manaus: Instituto de Pesquisas Ecológicas, 2010, 195 p.

SILVA, C. J.; SANCHES, L.; BLEICH, M.E.; LOBO, F.A.; NOGUEIRA, J.S. Produção de serrapilheira no Cerrado e Floresta de transição Amazônia-Cerrado do centro-oeste brasileiro. Acta Amazônica, v.37, n.4, p. 543-548, 2007.

SILVA, C.J.; LOBO, F.A.; BLEICH, M.E.; SANCHES, L. Contribuição de folhas na formação da serrapilheira e no retorno de nutrientes em floresta de transição no norte de Mato Grosso. Acta Amazonica, v. 39, n. 3, p. 591-600, 2009.

SILVA, D. U.; LOPES, M. S. Geografia, história e meio ambiente: a degradação ambiental em Alta Floresta / MT. Revista Científica Multidisciplinar Núcleo do Conhecimento, v.8. Ano 1. p. 144- 177, 2016.

SILVA, J. N. M.; CARVALHO, J. O. P.; LOPES, J. C. A.; ALMEIDA, B. F.; COSTA, D. H. M., OLIVEIRA, L. C.; et al. Growth and yield of a tropical rain forest in the Brazilian Amazon 13 years after logging. Forest Ecology and Management, v.71, p. 267-274, 1995.

SILVA, L. B. Relações entre aporte de serrapilheira, nutrientes e efluxo de dióxido de carbono em floresta inundável de Vochysia divergens Pohl no Pantanal Mato-Grossense. Tese (doutorado) - Universidade Federal de Mato Grosso, Instituto de Física, Programa de Pós-Graduação em Física Ambiental: Cuiabá, 2013. 
SOARES, R. V.; BATISTA, A. C.; TETTO, A. F. Meteorologia e climatologia florestal. Curitiba, 2015, $215 \mathrm{p}$.

SIMONELLI, M.; REIS, B. N.; HARB, T. B.; CORREIA, G. G. S. Produção de serapilheira como bioinidicador de restauração no município de Santa Leopoldina, ES. Anais... In: 1 을 Congresso Brasileiro de Reflorestamento Ambiental, 2011, Guarapari-ES.

SOLÓRZANO, A.; PINTO, J. R. R; FELFILI, J. M; HAY, J. D. V. Perfil florístico e Estrutural do componente lenhoso em seis áreas de cerradão ao longo do bioma Cerrado. Acta Botanica Brasílica, v. 26, n. 2, p. 328-341, 2012.

SOUSA, M. M. A produção de serrapilheira em uma consequência de 10 anos em uma floresta urbana: resultantes ecológicas da transformação da paisagem no Maciço de Pedra Branca, Rio de Janeiro. 2015. 107p. Dissertação de Mestrado (Pós-graduação em Geografia). Pontifícia Universidade Católica do Rio de Janeiro, Rio de Janeiro-RJ. 2015.

SOUZA, A. P. de; MOTA, L. L. da; ZAMADEI, T.; MARTIM, C. C.; ALMEIDA, F. T. de; PAULINO J. Classificação climática e balanço hídrico climatológico no estado de Mato Grosso. Revista Nativa, v. 01, n. 01, p.34-43, 2013.

VALADÃO, M. B. X.; MARIMON JUNIOR, B. H; OLIVEIRA, B.; LUCIO, N.W.; SOUZA, M. G. R.; MARIMON, B. S. Biomass hyperdynamics as a key modulator of forest self-maintenance in a dystrophic soil in the Amazonia-Cerrado transition. Sciencia Forestalis, v.44, n.110, p.475-485, 2016.

VALENTINI, C. M. A. Efluxo de $\mathrm{CO}_{2}$ do solo de uma área de floresta de transição no noroeste de Mato Grosso. 2004. 81 f. Dissertação (Mestrado em Física e Meio Ambiente) - Universidade Federal de Mato Grosso, Cuiabá-MT. 2004.

VALENTINI, C. M. A.; SOARES, G. S.; SANTANA, R. A.; GUIMARÃES, A. F. S.; SILVA, A. H. B. Produção, acúmulo e decomposição de serapilheira em uma área revegetada do Parque Estadual Massairo Okamura em Mato Grosso. Revista Holos, Ano 30, v.5, p. 211-221, 2014.

VALLS, J. F. M.; POZZOBON, M. T.; FÁVERO, A. P. Diversidade genética no estrato herbáceo da vegetação do Pantanal. Anais...In: 54을 CONGRESSO NACIONAL DE BOTÂNICA, 2003, Belém, PA. p. 161-163.

VIDAL, M. M.; PIVELLO, V. R.; MEIRELLES, S.T.; METZGER, J. P. Produção de serapiIheira em Floresta Atlântica secundária numa paisagem fragmentada (Ibiúna, SP): importância da borda e tamanho dos fragmentos. Revista Brasileira de Botânica, v.30, n.3, p.521-532, 2007.

VITAL, A.R.T.; GUERRINI, I.A.; FRANKEN, W.K.; FONSECA, R.C.B. 2004. Produção de serapilheira e ciclagem de nutrientes de uma Floresta Estacional Semidecidual em zona ripária. Revista Árvore, v. 8, p.793-800, 2004.

VITOUSEK, P. M.; SANFORD JUNIOR, R. L. Nutrient cycling in moist tropical forest. Annual Review on Ecology Systematics, v.17, p. 137-167, 1986. 
WALTER, B. M. T. Fitofisionomias do bioma Cerrado: síntese terminológica e relações florísticas. Doutorado em ecologia, Universidade de Brasília, 2006.

XU, X. N. HIRATA, E. Forest floor mass and litterfall in Pinus luchuensis plantations with and without broad-leaved trees, Forest Ecology and Management,v. 157, p. 165-173, 2002.

ZEILHOFER, P.; SCHESSL, M. Relationship between vegetation and environmental conditions in the Northern Pantanal of Mato Grosso, Brazil. Journal Biogeography, v. 27 , n. 1 , p. $159-168,2000$. 